We feel this work demonstrates the care needed when ascribing products to a catalytic reaction.

We thank Mr. D. I. Glass for assistance with tho experimental work.

C. C. MCCarn

G. W. GoDIN

The IDistillers Company, Ltd.,

Research Department,

Creat Burgh, Epsom,

Surrey.

I Shalya, V. V., Piontkovskaya, M. A., and Polyakov, M. Y., Ukrain. Khim Zhur., 27, 184 (1961).

"Wladow, D., Z. anorg. allgem. Chem., 27\%, 287 (1954)

${ }^{3}$ Yopova, X. I., and Latyshev, V. P., Doklady Akad. Nauk S.S.S.R., 147,

- Veatch, F., Callahan, J. L., Milberger, E. C., and Foreman, R. W., Proc. second Intern. Cong., Catalysis, Paris, 2, 2647 (1960)

Gullen, J. D., and Skirrow, G., Proc. Roy. Soc., A, 244, 312 (1958).

\section{Nuclear Magnetic Resonance in 'Monocrystalline' Polyoxymethylene}

General equations have been derived for the second moment of a nuclear magnetic resonanco line in an oriented polymor $^{1}$ on the basis of the Van Vleck formula ${ }^{2}$. These equations were verified for 'monocrystalline' polyoxy. mothylene obtained from a trioxane monocrystal by solidphase radiation-induced polymerization.

Monocrystals of polyoxymethylene, 5-6 $\mathrm{mm}$, were placed into a thin-walled glass ampoule in a way that their axes were normal to that of the ampoule. Nuclear mag. netic resonance spectra were obtained at $77^{\circ} \mathrm{K}$ at various angles, $\theta^{\prime}$, between the crystal axis and the magnetic field. A polycrystalline sample-small crystals disorderly packed into an ampoule-was prepared along with the monocrystals. The second moment value for the nuclear magnetic resonance line $\Delta \mathrm{H}_{2}^{2}=18.5 \pm 0.5 \mathrm{G}^{2}$ was determined for this sample. The $\Delta \mathbf{H}_{2}^{2}$ value was calculated theoretically for a known polyformaldehyde structuro ${ }^{3}$ and appeared to be $19 \cdot 9 \mathrm{G}^{2}$. The discrepancy of $1.5 \mathrm{G}^{2}$ is somewhat in excess over the experimental error and may be considered as due to deviation of the $\mathrm{C}-\mathrm{H}$ separation in the $\mathrm{CH}_{2}$-group from the value of $1.09 \AA$

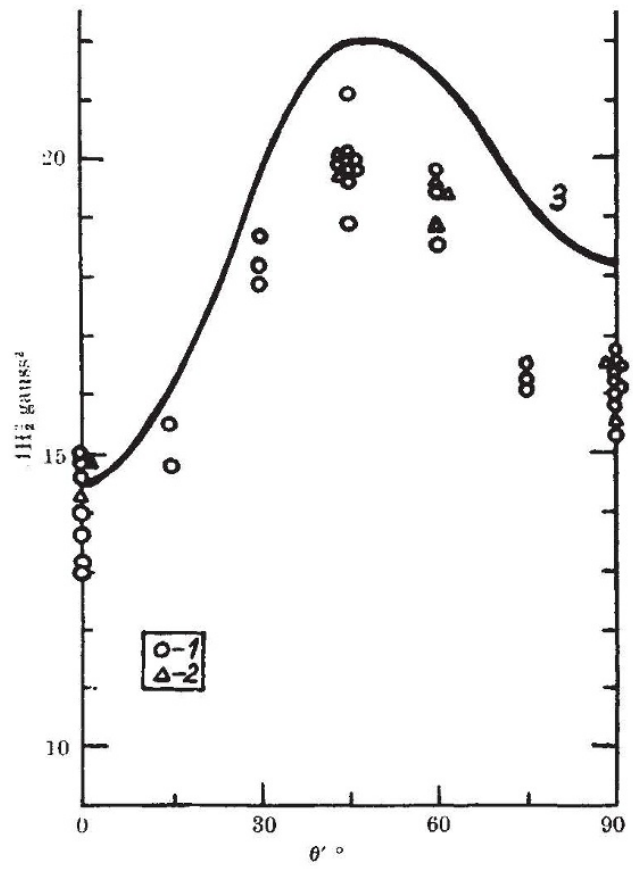

Fig. 1. Second moment of the nuclear magnetic resonance line $\left(\Delta \mathrm{H}_{2}^{2}\right)$ as a function of the angle $\theta$ ' between the 'monocrystal' axis and the magnetic field. 1 and 2, Experimental values for two samples; 3 , that was taken for calculation, or of the $\mathrm{H}-\mathrm{C}-\mathrm{H}$ angle from the tetrahedric form, or else to conservation of a enrtain degreo of molecular motion in the polymer at the liquid nitrogen temperature. The $\Delta \mathrm{H}_{2}^{2}$ value as a function of $\theta^{\prime}$ is shown in Fig. 1 for 'monocrystalline' samples. It was ascertained by X-ray analysis ${ }^{4}$ that polyoxymethylene 'monocrystals' obtained by radiation-induced polymerization consist in reality of crystallites of a dual orientation: the crystallite axes either coinciding with that of the macroscopic crystal, or inclined to it at an angle of $76^{\circ} 7^{\prime}$. The theoretical $\Delta \mathrm{H}_{2}^{2}-\theta^{\prime}$ function was calculated for this structure, allowance being made for contributions from intra- and inter-molecular proton interaction. For proton separations greater than $5 \AA$, summation in the Van Vleck formula was replaced by integration. It will be seen from Fig. 1 that tho calculated $\Delta \mathrm{H}_{2}^{2}$ values are essentially close to experimental ones. Yet they are somewhat higher than the latter: by $0.5 \mathrm{G}^{2}$ at $\theta^{\prime}=0$ and by $2 \mathrm{G}^{2}$ at $\theta^{\prime} \geqslant 45^{\circ}$. As with the polycrystalline sample, the diserepancy may bo partially due to inaccuracy of calculation. But molecular motion seems to be the main reason for it. Increase in the difference between theoretical and experimental $\Delta \mathrm{H}_{2}^{2}$ values with increasing $\theta^{\prime}$ might be an indication of a nonisotropic nature of molecular motion resulting in a extain narrowing of the nuclear magnetie resonanee lin".

\section{B. Netman f. Ya. SLONIM YA. (․ URMAN}

Institute of Chemical Physics,

U.S.S.R. Academy of Sciences, Institute of Plastics.

Moseow.

'Slonim, I. Ya., and Urman, Ya. G., Zhur. Strukt. Khim., 4, 216 (1963).

¿Van Vleck, J. H., Phys. Rev., 74, 1168 (1948).

${ }^{3}$ Tadokoro, H., Yasimoto, T., Murahashi, S., and Nitta, J., J. Poly. Sci., 44, $266(1960)$

" Carazzolo, G., Leghissa, S., and Mammi, M., Makromol. Chem., 60, 171 (1963).

\section{Thermal Helix-coil Transition in Poly- $\gamma$ - benzyl-L-glutamate}

THE thermally induced transition of solvated poly- $\gamma$ benzyl-L-glutamate (PBG) molecules from a helical to a random coil configuration has been the subject of recent investigations ${ }^{1-3}$. Similar transitions, brought about by temperature or by other changes, occur in several polyamino-acids ${ }^{4}$, and the general phenomenon has been treated theoretically by a number of authors ${ }^{5}$.

One of the results which emerges from such treatments is that under certain limiting conditions the transition may be regarded as a co-operativo first order change, analogous in some ways to melting. Experimentally it is found, however, that the transition is 'smeared' over a number of degrees and that an observable helix-random coil equilibrium exists in the vicinity of the transition temperature, $T_{c}$. By the application of the van't Hoff equation to this equilibrium an apparent heat of transition, $\Delta H$, may be calculated. In the formulation of Zimm and $\mathrm{Bragg}^{3}$ and of Apploquist ${ }^{6} \Delta H$ is related to the true heat of transition $\Delta H_{0}$ by the equation:

$$
\Delta H=\frac{\Delta H_{0}}{\sigma^{1 / 2}}
$$

where $\Delta H_{0}$ is the heat associated with the transfer of one amide residue from a random coil configuration to the end of an existing helical section, and $\sigma$ is a parameter related to the average length of the helical sertions at $T_{c}$. Zimm. Doty and $\mathrm{Iso}^{3}$ have provided an estimate of $2 \times 10^{-4}$ for $\sigma$ in the $\mathrm{PBG}$-dichloroacetic acid (DCA)-dichloroethane (DCE) system from an examination of the effect of chainlength on the shape of the transition curve, and thus, using equation $1, \Delta H_{0}$ may be derived. 